\title{
MERCURY-CONTAMINATED FISH AND ESSENTIAL FATTY ACIDS: PROBLEMS AND SOLUTIONS
}

\author{
Cropotova Janna, Popel Svetlana \\ Practical Scientific Institute of Horticulture and Food Technology, MD 2070 Chisinau, Codru town, str. Vierul, 59 \\ E-mail: janna_cr@mail.ru, phone number:+373 (022) 24-55-92, fax number:+373 (022) 24-16-88 \\ E-mail: sspopeli@mail.ru, phone number: +373 (022) 24-55-92, fax number: +373 (022) 24-16-88
}

\begin{abstract}
Fish consumption is an important part of human diet due to essential omega-3 fatty acids found naturally in this product. Many researchers from all over the world found that high mercury concentrations in the body reduced the heart-protective effects of the fatty acids in fish oils. People shouldn't be constrained by choosing between the health hazards related to toxins caused by industrial pollution and the nutritional benefits provided by consummation of essential fatty acids contained in oily fish. It is very important to find an alternative natural source of essential omega-3 fatty acids EPA and DHA to restore an optimal ratio between omega- 6 and omega- 3 fatty acids in the human diet. The Laboratory of Food Additives of the Practical Scientific Institute of Horticulture and Food Technology focuses on developing new essential vegetable oil blends with optimal ratio between omega- 6 and omega- 3 fatty acids.
\end{abstract}

Keywords: pollution, fish, mercury, fatty acids

\section{Introduction}

Health benefits of fish are linked to the essential nutrients such as omega-3 fatty acids, vitamins $\left(\mathrm{D}, \mathrm{B}_{2}\right.$, etc.) macro- and microelements such as calcium, phosphorus, iron, zinc, iodine, magnesium, and potassium. Two essential omega-3 fatty acids EPA (eicosapentaenoic acid) and DHA (docosahexaenoic acid) aren't produced by the human body and are found in every kind of fish, especially in fatty fish. Fish do not actually produce omega-3 fatty acids, but instead accumulate them by consuming either microalgae or prey fish that have accumulated omega- 3 fatty acids, together with high quantity of antioxidants as iodine and selenium, from microalgae, where these antioxidants are able to protect the fragile polyunsaturated lipids from peroxidation [1,2,3]. Practically all fish contain trace amounts of methyl mercury. As mercury moves up the food chain, it accumulates. As a result, larger predator fish, such as swordfish or tuna, tend to contain higher levels of mercury than salmon and other fish farther down the food chain. For this reason, the U.S. Food and Drug Administration recommends limiting consumption of certain predatory fish species due to high levels of toxic contaminants [4].

Organic mercury is most commonly found in the environment as methyl mercury: $\mathrm{CH}_{3} \mathrm{Hg}^{+}$, or $\mathrm{MeHg}$. Natural sources of elemental mercury include: degassing of the earth's crust, geologic deposits of mercury, forest fires and volatilization from the ocean. Levels of elemental mercury in the environment increase due to human industry: burning of fossil fuels, especially coal-burning plants, chemical industries, world-wide mining of gold and mercury. Once in water, mercury enters a complex cycle in which one form can be converted to another. Aquatic anaerobic bacteria identified as Enterobacter aerogenes convert elemental mercury to methyl mercury, which is taken-up by other microorganisms. Fish absorbs the toxic methyl mercury (from these microorganisms or other fish with concentrated $\mathrm{MeHg}$ ) as they feed in these contaminated waters, and so the toxin builds up in them.

There is concern about mercury contamination through fish all around the world, but specific recommendations vary. For example, Health Canada advises consumers to limit their consumption of swordfish, shark or fresh and frozen tuna to one meal per week; for young children and women of child-bearing age, the recommended limit is one meal per month. Health Canada's guideline is $0.5 \mathrm{ppm}$ total mercury content [5].

The FDA has released guidelines for children, women who are pregnant and women who are trying to become pregnant. These guidelines state that no more than $12 \mathrm{oz}$ of low mercury fish should be consumed weekly. "Highest" mercury fish should be avoided and "high" mercury fish should be kept to only three 6-oz servings per month [6].

Eating fish high in mercury puts middle-aged men at a greater risk for coronary heart disease and may offset the protective effects of omega- 3 fatty acids in some seafood, according to an important new Finnish study. The findings were based on an ongoing 14-year study by the Research Institute of Public Health at the University of Kuopio of 1,871 men ages 42 to 60 and free of previous heart disease or stroke [7].

The purpose of this paper is to determine balanced fatty acid profile using computer-assisted analysis of vegetable oil composition in accordance with WHO guidelines for further elaboration of the oil blends with optimal ratio of omega-3 and omega- 6 fatty acids, through the replacement of "high mercury" fish consumption with intake of essential vegetable oil blends. 


\section{Results and Discussion}

Computer-assisted analysis was used to identify nutritionally beneficial blends of commercially available edible oils, and to verify that their inclusion in alimentation is compatible with all current nutritional recommendations (WHO).

Computer-assisted analysis through Microsoft Excel by using Pearce's rule allows the translation of nutrient recommendations of polyunsaturated fatty acids into vegetable oil combinations. The preliminary forecasts have been developed through detailed calculations of experimental data from literary sources.

Fatty acid composition of some vegetable oils [8]

Table 1

\begin{tabular}{|c|c|c|c|c|c|}
\hline \multirow{2}{*}{ Nr. } & \multirow{2}{*}{ Vegetable oils } & \multicolumn{3}{|c|}{ Fatty acid composition (expressed as percentage of total acids) } & \multirow{2}{*}{$\begin{array}{c}\text { Ratio (calculated) } \\
\text { between } \\
\omega-3 \text { and } \omega-6\end{array}$} \\
\hline & & linolenic acid $(\omega-3)$ & linoleic acid $(\omega-6)$ & oleic acid ( $\omega-9)$ & \\
\hline 1 & Sunflower oil & $* \mathrm{ND}-0,3$ & $48,3-74,0$ & $14,0-39,4$ & $<0,004$ \\
\hline 2 & Rapeseed oil & $5,0-13,0$ & $11,0-23,0$ & $8,0-60,0$ & $0,45-0,56$ \\
\hline 3 & Maize oil & $\mathrm{ND}-2,0$ & $34,0-65,6$ & $20,0-42,2$ & $<0,03$ \\
\hline 4 & Soybean oil & $4,5-11,0$ & $48,0-59,0$ & $17,0-30,0$ & $0,09-0,19$ \\
\hline
\end{tabular}

*ND - non detectable, defined as $0,05 \%$

The most adequate resultant blends of vegetable oils with optimal ratio of omega- 3 and omega- 6 fatty acids were next: a) $89 \%$ sunflower oil with $11 \%$ flaxseed oil; b) $80 \%$ sunflower oil with $20 \%$ flaxseed oil; c) $62,5 \%$ sunflower oil with $37,5 \%$ rapeseed oil; d) $69 \%$ maize oil with $31 \%$ rapeseed oil; e) $35 \%$ sunflower oil with $65 \%$ soybean oil.

The present results, obtained using a simple computer-assisted analysis, confirm our suggestion that not single oil provides the recommended amounts and balance of essential fatty acids, but specific blends of vegetable oils in the proper proportions.

\section{Conclusion}

To resolve the problem associated with nutritional advantages of fish consumption while reducing exposure to mercury and keeping optimal ratio of essential fatty acids, it's necessary to replace "high mercury" fish consumption to vegetable oil blends and to consume mainly types of fish low in mercury, non-contaminated.

The consumption of essential oil blends composed of several vegetable oil in adequate proportion which can provide optimal ratio between omega- 6 and omega- 3 fatty acids is an inexpensive and simple way to meet current dietary recommendations for polyunsaturated fatty acids, favoring overall dietary nutrient requirements. Combination of vegetable oils instead of single oil is more likely to achieve current nutritional recommendations. Such combinations are relatively easy to create via compositional modifications to improve the nutritional value of vegetable oils used for seasoning.

\section{References}

[1]. Venturi S, Donati FM, Venturi A, Venturi M (2000). "Environmental iodine deficiency: A challenge to the evolution of terrestrial life?". Thyroid 10 (8): 727-9.

[2]. Venturi S, Venturi M (2007). «Evolution of Dietary Antioxidant Defences». European Epi-Marker 11 (3): 1-12.

[3]. Küpper FC, Carpenter LJ, McFiggans GB, et al. (2008). "Iodide accumulation provides kelp with an inorganic antioxidant impacting atmospheric chemistry". Proceedings of the National Academy of Sciences of the United States of America 105 (19): 6954-8.

[4]. FDA/EPA Fish Consumption Advisory: EPA-FDA Joint Federal Advisory for Mercury in Fish. Retrieved 200702-08.

[5]. Food safety facts on mercury and fish consumption. Ottawa: Canadian Food Inspection Agency, May 2002.

[6]. FDA, Centre for Food Safety and Applied Nutrition (March 2001). Advisory: An Important Message for Pregnant Women and Women of Childbearing Age Who May Become Pregnant About the Risks of Mercury in Fish.

[7]. Tiina Rissanen, Sari Voutilainen, Kristiina Nyyssönen, Timo A. Lakka, Jukka T. Salonen. Fish Oil-Derived Fatty Acids, Docosahexaenoic Acid and Docosapentaenoic Acid, and the Risk of Acute Coronary Events. Research Institute of Public Health, University of Kuopio, Kuopio, Finland. < http://circ.ahajournals.org/ content/102/22/2677.full>

[8]. CODEX STANDARD 210 - 1999 FOR NAMED VEGETABLE OILS 